\title{
Effect of Bisphenol A-induced Oxidative Stress on the Ultra Structure and Antioxidant Defence System of Arabidopsis thialiana Leaves
}

\author{
Imran Ali ${ }^{1,2}$, Abdul Wakeel', Sakila Upreti' ${ }^{1}$, Dongdong Liu' ${ }^{1}$, Azizullah Azizullah ${ }^{3}$, \\ Mehmood Jan', Waheed Ullah², Bohan Liu', Abid Ali' ${ }^{1}$, M.K. Daud ${ }^{1}$, Yinbo Gan'* \\ 'Zhejiang Key Lab of Crop Germplasm, Department of Agronomy, College of Agriculture and Biotechnology, \\ Zhejiang University, Hangzhou, China \\ ${ }^{2}$ Department of Biotechnology and Genetic Engineering, Kohat University of Science and Technology, \\ Kohat, Pakistan \\ ${ }^{3}$ Department of Botany, Kohat University of Science and Technology, Kohat, Pakistan
}

Received: 7 March 2017

Accepted: 23 July 2017

\begin{abstract}
Bisphenol A (BPA) is an emerging environmental pollutant with potentially toxic effects on living organisms. The present study was undertaken to analyze the effects of BPA on the leaves of Arabidopsis thialina by determining the levels of photosynthetic pigments, reactive oxygen species (ROS), membrane lipid peroxidation, and ultrastructural malformation. The obtained results revealed that while a low dose of BPA $(10 \mu \mathrm{M})$ did not alter the test indices significantly, it did cause significant changes in all test indices at higher concentrations. Upon exposure to $40 \mu \mathrm{M}$ BPA, chlorophyll $a$ and chlorophyll $b$ content showed a decrease of $33 \%$ and $30 \%$, respectively. It significantly increased ROS contents and lipid peroxidation at $40 \mu \mathrm{M}$ BPA exposure. Biochemical and gene expression analysis revealed that the antioxidant system was activated and mounted a defense against BPA-induced ROS. In the case of superoxide dismutase (SOD), $40 \mu \mathrm{M}$ of BPA caused an increase of $151 \%$. However, the malfunctioning of ascorbate peroxidase (APX) and catalase $(\mathrm{CAT})$ at the highest dose of BPA $(40 \mu \mathrm{M})$ resulted in incomplete activation of the antioxidant defensive system. BPA stress significantly altered the ultrastructure of cells as evidenced by the reduced number of starch grains, damaged chloroplast and mitochondria, and altered leaf epidermal surface, guard cells, and stomata. It is concluded that observed adverse effects in Arabidopsis leaves in response to BPA exposure could be attributed to BPA-induced oxidative stress.
\end{abstract}

Keywords: bisphenol A, antioxidant, ultrastructure, oxidative stress, ROS

*e-mail: ygan@zju.edu.cn 


\section{Introduction}

Plants often grow in environments where they are exposed to a variety of biotic and abiotic stresses. These stresses can hinder plants from attaining their full genetic potential that is necessary for growth and reproduction [1]. Plants respond to abiotic and biotic stresses through a series of complex mechanisms involving changes at physiological, cellular, and transcriptomic levels [2]. By adopting these survival mechanisms plants may avoid damage and ensure their growth and development under adverse environmental conditions, but at the expense of reduced growth and yield [3].

Bisphenol A (BPA; 2, 2-bis-(4- hydroxyphenyl) propane) is a key raw material in the production of phenol resins, transparent polycarbonate plastic, poly-acrylates, and polyesters [4]. Due to its wide use in the production of industrial and household items, the global production of BPA is at an unprecedented high. It has been estimated that the global annual production of BPA is 6.8 million tons [5], and the annual growth rate of demand is $6 \%$ to $10 \%$ [6]. As a result of its massive usage, BPA has been continuously brought into the environment through industrial waste, sewage sludge, and waste landfill leachate [6]. In the Netherlands and Germany, BPA concentrations up to 21 $\mu \mathrm{g} / \mathrm{Land} 0.4 \mu \mathrm{g} / \mathrm{L}$, respectively, were detected in surface water [5]. In Japan, the content of BPA in garbage landfill leachate was found to reach $17.2 \mathrm{mg} / \mathrm{L} \mathrm{[7]}$. Such relentless emissions of BPA into the environment has seriously compromised the environmental safety and balance of the ecosystem [8] it is now clear that an understanding of the effects of the environment on reproduction requires a far broader range of expertise and that, at least for endocrine-disrupting chemicals, many of the tenets of classical toxicology need to be revisited. Indeed, because of the wide range of reproductive effects induced by these chemicals, interest among reproductive biologists has grown rapidly: in 2000, the program for the annual Society for the Study of Reproduction meeting included a single minisymposium on the fetal origins of adult disease, one platform session on endocrine disruption, and 23 toxicology poster presentations. In contrast, environmental factors featured prominently at the 2009 meeting, with strong representation in the plenary, minisymposia, platform, and poster sessions. Clearly, a lot has happened in a decade, and environmental issues have become an increasingly important research focus for reproductive biologists. In this review, we summarize some of the inherent difficulties in assessing environmental effects on reproductive performance, focusing on the endocrine disruptor bisphenol A (BPA. Several studies have repeatedly confirmed the toxicological effects of BPA on humans and animals. BPA has been reported to cause physiological disorders, damage reproductive and endocrine systems, and alter genetic materials [9-11].

With increasing population water scarcity is becoming a serious global problem. Due to the limitation of water resources and lack of water management strategies [12-
13], the use of sewage and sludge water for irrigation purposes is on the rise. Although some important aspects of irrigation in agriculture water management have been extensively investigated in previous work [14-16], limited information is available regarding irrigation of agricultural land using polluted water. Therefore, BPA is becoming ubiquitous in soil due to the widespread and continuous use of sewage and sludge water for irrigation [17]. BPA is ubiquitous in soil due to wide and continuous use of sewage and sludgewater for irrigation [17]. The addition of wastewater treatment plant bio-solids (a byproduct from municipal wastewater treatment plants) to soil for the amendment of agricultural land is also a major cause of BPA in soil [17]. Although BPA is readily biodegraded by microorganisms, its residues in soil can remain for some time [3]. It has been reported that rooted plants, e.g., tobacco, can take up and transport BPA to the aboveground parts after metabolizing it to a non-toxic metabolite BPA glycosides [18]. However, there is enough evidence that BPA can adversely affect plant growth and development by interfering with key physiological and metabolic processes.

The effects of BPA on animals and humans have been frequently reported, but the available literature regarding the effects of BPA on plants is limited. The few available studies have revealed that BPA can adversely affect plants, but these studies have mainly been confined to the effects of BPA on seed germination, seedling growth, plant morphology, photosynthesis, BPA uptake, translocation, and accumulation [18-20], and have rarely focused on the mechanism of BPA toxicity in plants. Previous studies also have revealed that different plants species may respond differently to BPA stress. For example, Ferrara et al. [21] reported that a $10 \mathrm{mg} / \mathrm{L}$ and $50 \mathrm{mg} / \mathrm{L}$ of BPA in a hydroponic culture significantly inhibited root-shoot length and fresh and dry biomass in tomato, durum wheat, and lettuce, but the same concentration of BPA did not alter these indices in broad beans. Other studies reported that at low concentration BPA stimulated different growth parameters in plants but significantly inhibited these parameters at high concentrations $[19,22]$. BPA has also been found to interfere with nitrogen metabolism and photosynthesis in plants [22].

The impact of BPA on plant growth and development involves biochemical disturbance mainly through oxidative stress [6]. The production of excess reactive oxygen species (ROS) such as superoxide radical $\left(\mathrm{O}_{2}^{-}\right)$and hydrogen peroxide $\left(\mathrm{H}_{2} \mathrm{O}_{2}\right)$ creates oxidative stress in chloroplasts, plasma membrane, mitochondria, and peroxisomes [23]. This exaggerated increase in ROS accumulation damages cell membranes and organelles, causes changes in vital physiological processes, and ultimately limits plant growth [24]. The accumulation of excess ROS triggers a complex ROS scavenging mechanism involving non-enzymatic and enzymatic systems to prevent uncontrolled oxidation [25]. The ROS scavenging mechanism in plants consists of some key enzymes such as superoxide dismutase (SOD), which catalyzes the conversion of $\mathrm{O}_{2}^{-}$to $\mathrm{H}_{2} \mathrm{O}_{2}$, which is further 
detoxified to $\mathrm{H}_{2} \mathrm{O}$ by the actions of APX, CAT, and POD enzyme [1].

Arabidopsis is one of the most important model plants and its use as a model system has been employed to study the complex network of factors involved in plant responses to environmental stresses. Furthermore, BPA contamination of soil and irrigation water may potentially damage cell organelles mainly through oxidative stress. However, the effect of BPA on Arabidopsis has never been investigated previously. In particular, there is no specific information available regarding the effects of BPA on the biochemical process and cell fine structures in the model plant Arabidopsis thialiana. Considering the wide range of plant responses to BPA exposure and the scarcity of studies regarding the toxicity of BPA to a model plant Arabidopsis thialiana, the present study was design to investigate the biochemical responses and ultrastructure alteration in leaves of Arabidopsis thialiana upon exposure to BPA.

\section{Materials and Methods}

\section{Plant Materials}

Seeds of Arabidopsis thialiana (Columbia ecotype) wild type ( $W T$ ) were surface sterilized in $5 \%$ (v/v) sodium hypochlorite for eight minutes, followed by five times washing with sterile distilled water, and stratified in the dark for three days at $4^{\circ} \mathrm{C}$. The sterilized seeds were grown hydroponically according to the modified method of [26] we found through trialling systems as they are described in the literature that our experiments were frequently confounded by factors that affected plant growth, including algal contamination and hypoxia. We also found the way in which the plants were grown made them poorly amenable to a number of common physiological assays. Results: The drivers for the development of this hydroponic system were: 1 in half-strength MS medium ( $\mathrm{pH}$ 5.8). The seedlings were grown in a controlled environment $\left(22^{\circ} / 18^{\circ} \mathrm{C}, 12 / 12 \mathrm{~h}\right.$ light/dark photoperiod, $75 \%$ relative humidity, $150 \mu \mathrm{mol} \mathrm{m} \mathrm{m}^{-2} \mathrm{~s}^{-1}$ photon flux) for two weeks. After two weeks, seedlings were subjected to BPA stress by transferring the seedlings to half MS medium supplemented with different dozes (0 (control), $10,20,30,40 \mu \mathrm{M})$ of BPA. The seedlings were harvested after five days of BPA exposure and samples were collected for further experiments.

\section{Measurement of Fresh Weight and Light Harvesting Pigments}

After five days of BPA exposure, fresh weigh of rosettes $(n=12)$ was determined in milligrams. For quantification of photosynthetic pigments five plants were randomly selected in each replicate. Photosynthetic pigments (chlorophyll $a$, chlorophyll $b$, and total carotenoids) were extracted in $80 \%$ acetone using the method described by Ahmmad et al. [27].

\section{Scanning Electron Microscopy (SEM)}

For scanning electron microscopy (SEM), leaf segments $(n=6)$ without veins were taken from control $\left(\begin{array}{ll}0 & \mu \mathrm{M}\end{array}\right)$ and $40 \mu \mathrm{M}$ BPA-treated seedlings and were immediately fixed in $2 \%$ glutaraldehyde in $0.05 \mathrm{M}$ sodium cacodylate buffer at $\mathrm{pH}$ 7.2. The samples were then put in $1.0 \%$ osmium tetroxide in $0.1 \mathrm{M}$ sodium cacodylate buffer at $\mathrm{pH} 7.2$ for $1 \mathrm{~h}$, followed by three washes with sodium phosphate buffer (PBS). For dehydration the samples were placed in a graded series of ethanol (30, $50,70,80,85,90,95$, and $100 \%$ ) for 15 minutes. The samples were then dried through liquid carbon dioxide and mounted in metal stubs and sputter-coated with 20 $\mathrm{nm}$ gold. The prepared samples were examined under a scanning electron microscope (LEO 435 VP, Cambridge, England).

\section{Transmission Electron Microscopy (TEM)}

For TEM analysis leaf segments $(n=6)$ without veins from untreated $(0 \mu \mathrm{M})$ and treated $(40 \mu \mathrm{M}$ BPA) seedlings were fixed in $4 \%(\mathrm{v} / \mathrm{v})$ gluteraldehyde in $0.1 \mathrm{M}$ sodium phosphate buffer ( $\mathrm{pH} 7.4)$. After $48 \mathrm{~h}$ the samples were washed three times with buffer followed by post-fixation in $1.0 \%$ osmium tetroxide for $2 \mathrm{~h}$ and then repeated washing with PBS. Afterward the samples were dehydrated in a graded series of ethanol $(30 \%, 50 \%$, $70 \%, 80 \%, 85 \%, 90 \%, 95 \%$, and $100 \%$ ) for 15 minutes each and then in absolute acetone for 20 minutes. The samples were then embedded in a spurr's resin at $70^{\circ} \mathrm{C}$ for $9 \mathrm{~h}$. Ultra-thin sections were made and mounted on copper grids and scanned using a transmission electron microscope (TEM 1230EX, JEOLK Japan) at $60.0 \mathrm{kV}$.

$$
\begin{aligned}
& \text { Lipid Peroxidation and Histochemical Detection } \\
& \text { of } \mathrm{H}_{2} \mathrm{O}_{2}, \mathrm{O}_{2}^{-} \text {in Leaves }
\end{aligned}
$$

Lipid peroxidation was determined in terms of thiobarbuteric acid reactive substances (TBARS) following the protocol of Gratao et al. [28].

The $\mathrm{H}_{2} \mathrm{O}_{2}$ accumulation in leaves of the control $(0 \mu \mathrm{M})$ and $40 \mu \mathrm{M}$ BPA-treated seedlings was determined by 3,3-diaminobenzidine (DAB) staining method as described by Thordal-Christensen et al. [29] Thordal-Christensen, Zhang [20]. The $\mathrm{O}_{2}^{-}$accumulation was determined by staining the leaves using nitro blue tetrazolium (NBT) using the protocol of Fryer et al. [30].

\section{Measuring Antioxidant Enzyme Activity}

For anti-oxidant enzyme analysis a $0.3 \mathrm{~g}$ fresh leaf sample was homogenized in $9 \mathrm{ml}$ of $50 \mathrm{mM}$ potassium phosphate buffer $(\mathrm{pH}$ 7.8) by grinding with a pre-chilled mortar and pestle under freezing conditions. The homogenate was centrifuged twice at $14,000 \mathrm{x}$ g for 15 minutes at $4^{\circ} \mathrm{C}$, and the supernatant was then stored at $-20^{\circ} \mathrm{C}$ and utilized for the determination 
of various antioxidant enzyme assays (SOD, POD, APX, and CAT).

Superoxide dismutase (SOD, EC 1.15.1.1) activity was measured using the protocol described by Daud et al. [31]. The activity of peroxidase (POD) (EC1.11.1.7) was determined by taking OD at $470 \mathrm{~nm}$ as previously described by [32]. The ascorbate peroxidase (APX) activity was measured according to [33]. The $3 \mathrm{ml}$ reaction mixture was comprised of $50 \mathrm{mM}$ potassium phosphate buffer ( $\mathrm{pH}$ 7.8), $0.1 \mathrm{mM}$ EDTA-Na, $0.3 \mathrm{mM}$ ascorbic acid, $0.06 \mathrm{mM} \mathrm{H}_{2} \mathrm{O}_{2}$, and $100 \mu \mathrm{l}$ of enzyme extract. The change in absorbance was measured at $290 \mathrm{~nm}$ after $30 \mathrm{~s}$ of the addition of $\mathrm{H}_{2} \mathrm{O}_{2}$.

Catalase (CAT, EC 1.11.1.6) activity was assayed according to the protocol of Aebi [34] by measuring $\mathrm{H}_{2} \mathrm{O}_{2}$ consumption (extinction coefficient, $39.4 \mathrm{mMcm} \_1$ ) for 1 minute at $\mathrm{A} 240$ in a $3 \mathrm{ml}$ reaction mixture containing $50 \mathrm{mM}$ potassium phosphate buffer $(\mathrm{pH} 7.0)$,

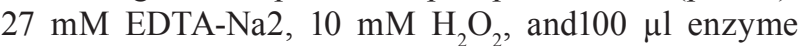
extract.

\section{Isolating Total RNA and Real-Time Quantitative (qRT-PCR) Expression Analysis}

The RNA samples from the leaves were isolated using RNAiso reagent (TaKaRa, Dalian, China) following manufacturer's instructions. The single stranded cDNA synthesis was performed using M-MLV reverse transcriptase (Promega) and oligold (T18) primers. Quantitative real-time PCR was carried out in an optical 96-well plate with a CFX96TM Real-time Detection System using the method described by Liu et al. [35]. The baseline and threshold cycles $\left(\mathrm{C}_{\mathrm{T}}\right.$ value $)$ were automatically determined by Roche Light Cycler 96 software (version 1.1.0.1320). Relative expression was calculated using the relative method of quantification, as the $2-\Delta \Delta \mathrm{C}_{\mathrm{T}}$ method described before Livak and Schmittgen [36].

\section{Data Analysis}

The plants were grown in three replicates with 20 plants per replicate (pot) and experiments were performed twice. Data were analyzed using IBM SPSS statistics 22 software and expressed as mean \pm standard error and standard deviation (q-PCR). Differences among the treated and untreated groups were compared by one-way analysis of variance (ANOVA) with Tukey's posthoc test at a significant level of $95 \%(\mathrm{P}<0.05)$.

\section{Results}

How BPA Exposure Reduced the Pigment Content in Arabidopsis leaves

Fig. 1a) shows the effects of BPA on photosynthetic pigments (chlorophyll $a$, chlorophyll $b$, and total carotenoids). There was no significant effect on chlorophyll $a$ at 10 and $20 \mu \mathrm{M}$ of BPA except for a slight and non-significant increase at $10 \mu \mathrm{M}$. However, at doses above $30 \mu \mathrm{M}$, BPA caused a significant decrease in chlorophyll $a$ content. In the case of chlorophyll $b$, no significant effect was observed except for a decrease at the highest tested concentration $(40 \mu \mathrm{M})$. The concentration of total carotenoids was significantly increased by BPA at the lowest tested concentration, but no significant effect was observed thereafter.

Fig. 1b) showed the effect of BPA on rosette fresh weight of Arabidopsis seedlings. Compared to control, at lower concentrations of BPA exposure no significant changes in rosette fresh weight were observed. However, at the highest tested concentration of BPA $(40 \mu \mathrm{M})$ the rosette fresh weight decreased significantly as compared to control.

\section{Expression Levels of Chlorophyll Synthesis Genes in BPA-treated Plants were Reduced}

Having shown that BPA exposure reduced the chlorophyll content in Arabidopsis, we decided to examine if the expression of the genes involved in chlorophyll synthesis are affected by BPA exposure (Fig. 1c). We examined the expression of several genes that have been reported to be involved in chlorophyll synthesis, including CHLOROPHYLLAOXYGENASE (CAO), CHLOROPHYLLSYNTHASE (CHLG), COPPER RESPONSE DEFECT 1 (CRD1), and MG-CHELATASE SUBUNITD (CHLD) [37-39]. The primers of these genes have been described previously [40]. As shown in (Fig. 1C), the expression of all the genes was increased upon exposure to lower concentrations of BPA (20 and $30 \mu \mathrm{M})$, however, at the highest BPA concentration the transcript level of CAO, CRDI, and CHLG genes decreased considerably as compared to the control. The expression level of $C H L D$ also decreased at the highest concentration of BPA, but remained higher as compared to the control.

\section{BPA Induces Structural Changes in Stomata and Guard Cells}

To determine if BPA exposure affects leaf epidermal surface, we analyzed the abaxial leaf surfaces of Arabidopsis in the control and BPA-treated samples (40 $\mu \mathrm{M})$ using scanning electron microscopy (SEM). The SEM images showed clear differences between the control and BPA-treated cells for pavement cells and stomata (Fig. 2). In the control samples, the pavement cells showed a characteristic jigsaw puzzle shape, but in BPA-treated leaves the pavement cells appeared to be deformed and overlapped each other, thus showing an irregular jigsaw puzzle shape. In BPA-treated leaves the stomata appeared deformed and irregular with wide openings, possibly due to shrinkage of guard cells.

\section{BPA Exposure-Altered Organelle Structure}

The effect of BPA on cellular structures, as revealed by electron micrographs of leaf sections, is shown in 
a)

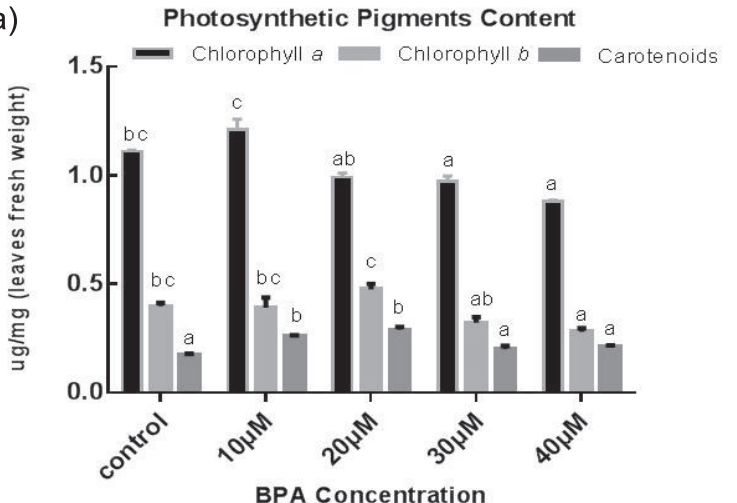

b)

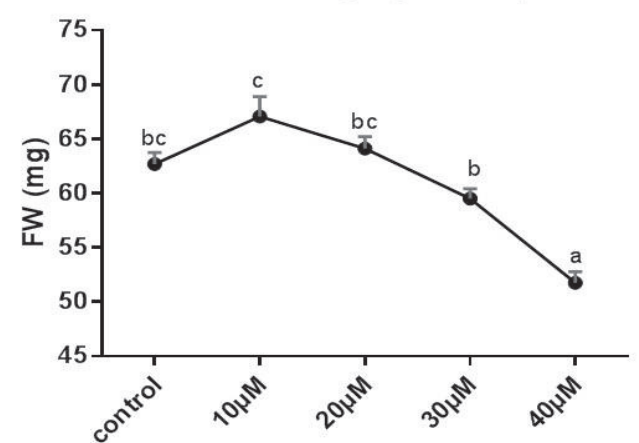

c)

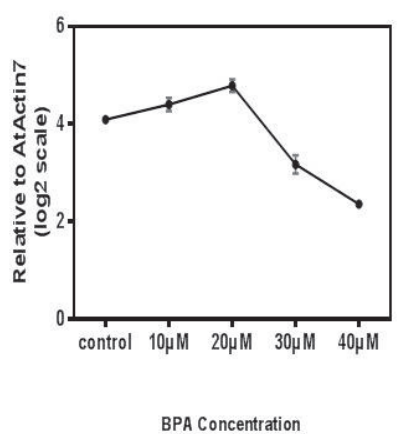

CRD1

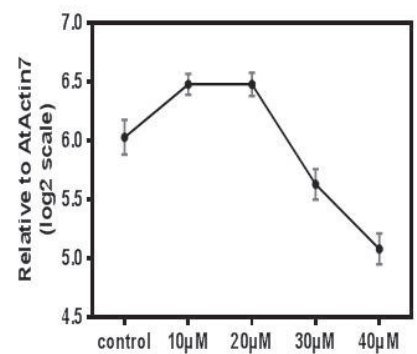

BPA Concentration

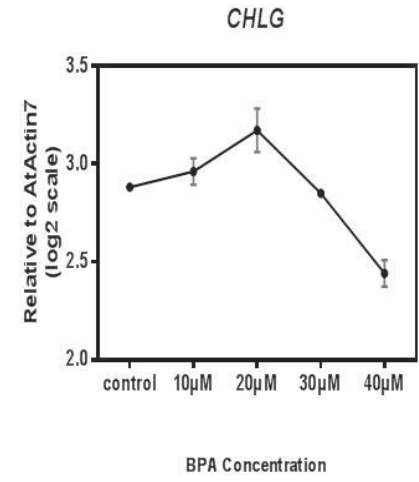

CHLD

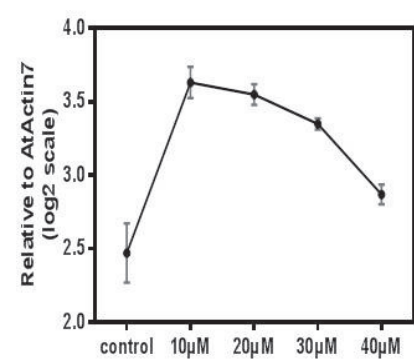

BPA Concentration

Fig. 1.a) Analysis of pigment content in $\operatorname{Arabidopsis}(W T)$ leaves in response to BPA exposure b), and the effect of BPA on rosette fresh weight $(n=12)$ of $W T$. The error bars indicate means \pm SE. Different letters indicate significant differences $(\mathrm{P}<0.05)$. c) Effect of BPA on the transcription level of genes related to chlorophyll synthesis in leaves of Arabidopsis (WT). The transcription levels were normalized with AtActin 7 in each sample, and the expression value was presented in $\log 2$ scale, error bar mean $\pm \mathrm{SD}$.
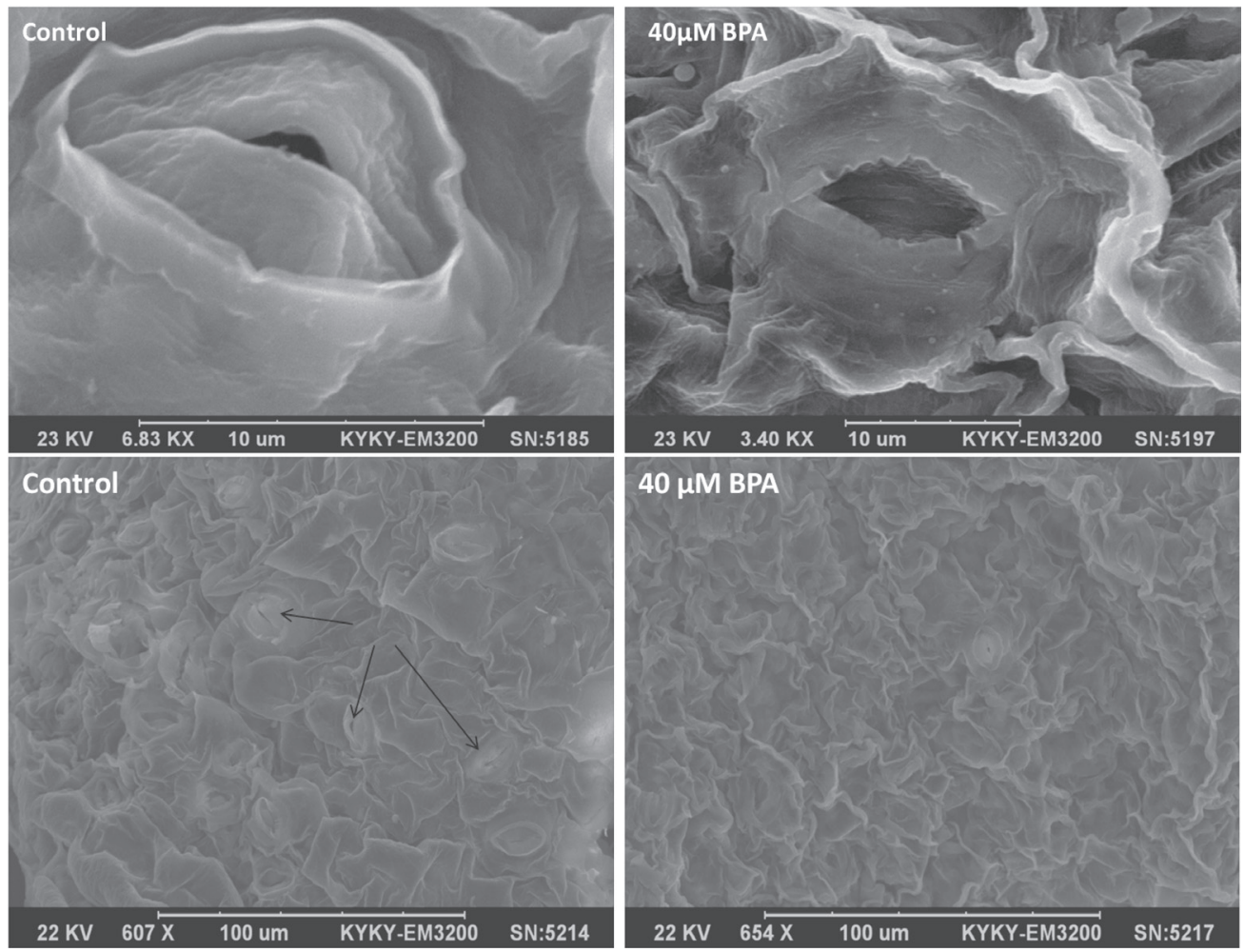

Fig. 2. Abaxial epidermis of Arabidopsis (WT) with or without $40 \mu \mathrm{M}$ BPA. Stomata and guard cells of Arabidopsis WT, plants with or without BPA (control). Black arrow signs represent open stomata in control samples. 

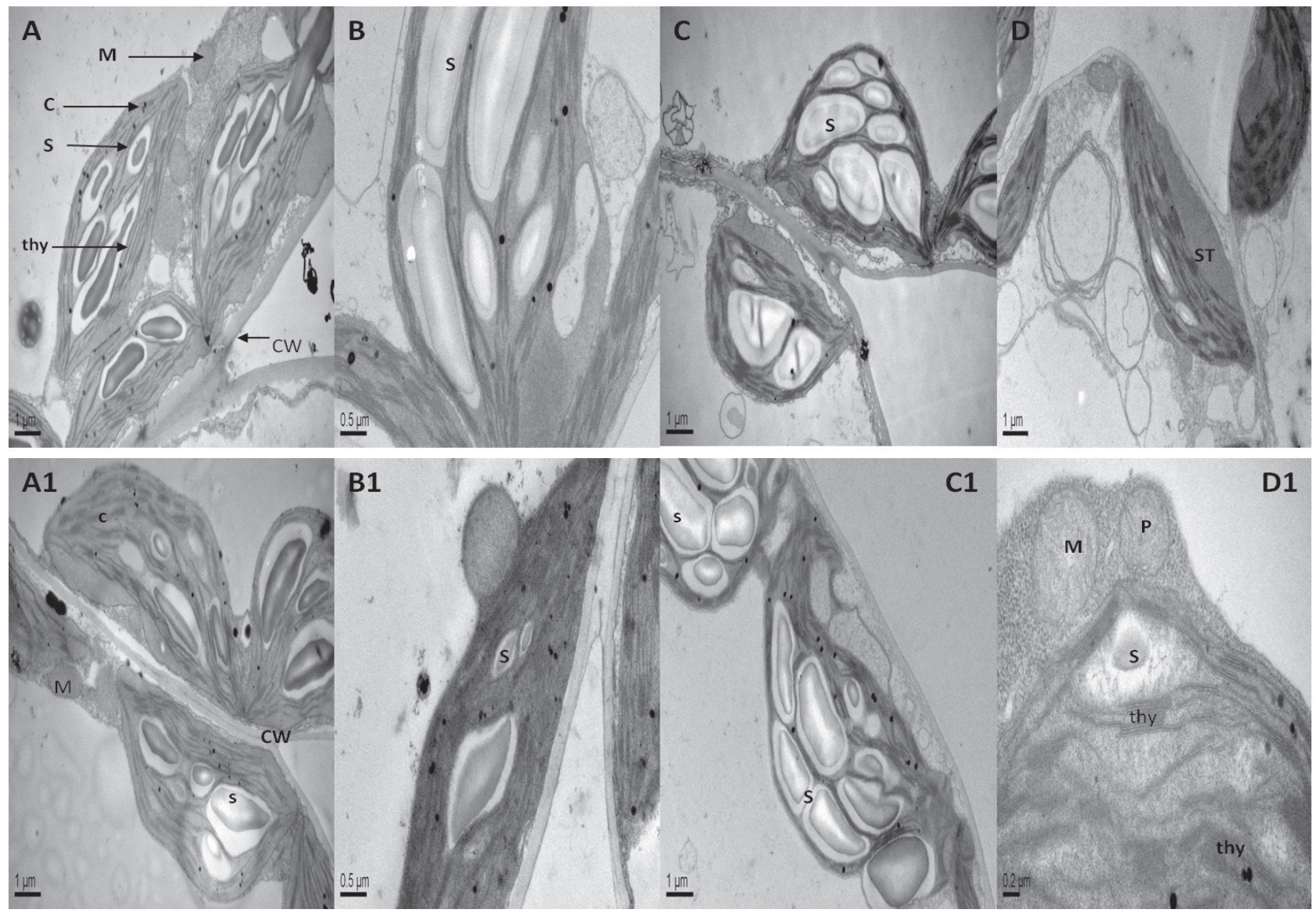

Fig. 3. Organelle ultrastructure of leaf mesophyll cells of Arabidopsis WT with or without $40 \mu \mathrm{M}$ BPA. (A, B, C, D) control leaf cells. (A1, B1, C1, and D1) leaf cells of $W T$-treated with $40 \mu \mathrm{M}$ BPA. $\mathrm{C}=$ chloroplast, thy $=$ thylakoid, $\mathrm{S}=$ starch grain, $\mathrm{CW}=$ cell wall, $\mathrm{M}=$ mitochondria, $\mathrm{P}=$ Perioxisomes

Fig. 3. In the control samples, typical structural features of organelle were clearly visible, where intact lens-shaped chloroplast with well-defined grana, thylakoid, stroma, and starch grains with a well-developed membrane system were observed (Fig. 3A-D). The control mitochondria were mainly oval shaped with well-defined cristae. The controlled chloroplasts were elongated and their length was at least twice the width. Although they have a well-defined envelope membrane (Fig. 4), in BPA-treated leaf cells very few chloroplasts were present and were mostly deformed with an abnormal shape and degraded chloroplast envelope (Figs 4A1 and 4C1). Moreover, BPA greatly damaged and reduced the number of starch grains as compared to untreated leaves. Similarly, BPA treatment adversely affected the thylakoid region, as evident by disrupted thylakoids in treated cells (Fig. 4B1). BPA treatment reduced the mitochondria count, deformed them, and disappeared the mitochondrial cristae. In BPA-treated cells an increased number of plastoglobule were also observed (Fig. 4B1). The main alterations observed in the leaves of BPA-treated cells were the disruption of chloroplast with disorganized grana and stroma and disarrangement of thylakoids (Figs 3A1, B1, C1, and D1).

\section{BPA Exposure Induces Lipid Peroxidation and ROS Generation}

Fig. 5b) shows the induction of lipid peroxidation in the leaves of Arabidopsis in response to BPA exposure determined in terms of thio-barbuteric acid reactive substances (TBARS). Exposure to BPA resulted in a dose-dependent increase in TBARS content, and this effect was statistically significant at the highest tested two concentrations (30 and $40 \mu \mathrm{M})$. Since TBARS are the end-product of lipid peroxidation, the measurement of $\operatorname{ROS}\left(\mathrm{H}_{2} \mathrm{O}_{2}\right.$ and $\left.\mathrm{O}_{2}^{-}\right)$is a suitable physiological index to explain the increase in TBARS. In the leaves of control seedlings, no considerable accumulation of $\mathrm{H}_{2} \mathrm{O}_{2}$ and $\mathrm{O}_{2}^{-}$was observed (Fig. 5a). However, a considerable increase in the accumulation of $\mathrm{H}_{2} \mathrm{O}_{2}$ and $\mathrm{O}_{2}^{-}$was shown with the increase in concentration of BPA (Fig. 4). At the highest tested concentration of BPA $(40 \mu \mathrm{M})$, more brown spots (for $\mathrm{H}_{2} \mathrm{O}_{2}$ ) and blue spots $\left(\mathrm{O}_{2}^{-}\right)$were observed, which indicated the generation and accumulation of excess ROS in response to BPA exposure. These observations correspond well to the pattern of TBARS increase with the increase in BPA concentration. 


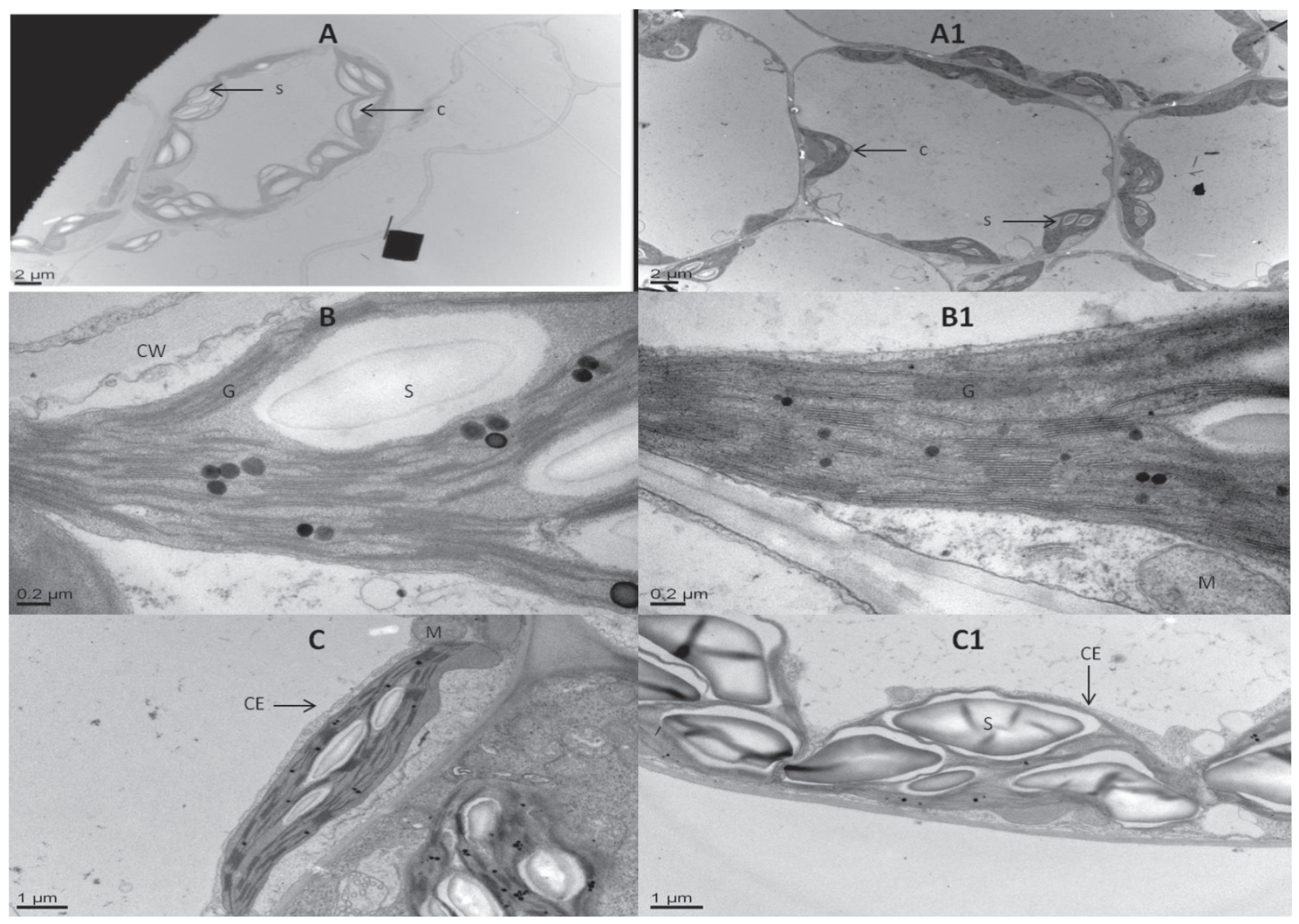

Fig. 4. Transmission electron micrographs of chloroplast with or without $40 \mu \mathrm{M}$ BPA. Control cells (A, B, C), treated cells (A1, B1, C1). $\mathrm{CE}=$ chloroplast envelop, thy $=$ thylakoid, $\mathrm{S}=$ starch grain, $\mathrm{M}=$ mitochondria, $\mathrm{P}=$ plastoglobule, $\mathrm{G}=$ grana

\section{Antioxidant Enzyme Activity Activated upon BPA Exposure}

Fig. 6a) shows the effects of bisphenol $\mathrm{A}$ on antioxidant enzyme activity in Arabidopsis leaves. As compared to the control, at $10 \mu \mathrm{Mof}$ BPA the activities of CAT, POD, and APX did not change significantly; however, the activities of SOD enzyme significantly increased. After exposure to $20 \mu \mathrm{M}$ of BPA, SOD activity significantly increased while a non-significant increase was observed in the activities of all other enzymes as compared to the control. A $30 \mu \mathrm{M}$ of BPA caused an increase in the activities all four antioxidant enzymes, but this increase was statistically significant only in the case of SOD and APX. At the highest tested concentration $(40 \mu \mathrm{M})$ of BPA, the activities of SOD and POD were significantly increased while CAT activity showed a significant and APX activity a nonsignificant decrease.

\section{Expression of Antioxidant Defense System Genes}

BPA exposure induced the expression of known antioxidant defensive system genes in Arabidopsis thialiana (Fig. 6b). The primers used for these genes have been described previously [41]. Transcripts of CAT-encoding genes CAT2 (AT4G35090) and CAT3 (AT1G20620) were increased by BPA exposure at lower concentrations. However, a significant decrease was observed in both CAT2 and CAT3 expression levels upon exposure to highest BPA concentrations $(40 \mu \mathrm{M})$. The expression pattern of superoxide dismutase was not very clear, as the abundance of $\mathrm{Cu} / \mathrm{Zn}$-SOD-encoding gene CSD1 (AT1G08830) increased upon BPA exposure, whereas the expression of $\mathrm{Mn}$-SOD-encoding gene $M S D$ (AT3G56350) decreased at $40 \mu \mathrm{M}$ BPA after an initial increase at lower concentrations of BPA. Transcripts of POD-encoding gene POD3 (AT1G05260) were increased by BPA at all concentrations. The transcript level of APXencoding gene APX1 (AT1G07890) was decreased by BPA exposure in comparison to the control.

\section{Discussion}

When plants encounter stressful conditions they devise an adaptation mechanism in order to withstand them. One such mechanism is altering their metabolism by changing physiological activities, which are regulated 


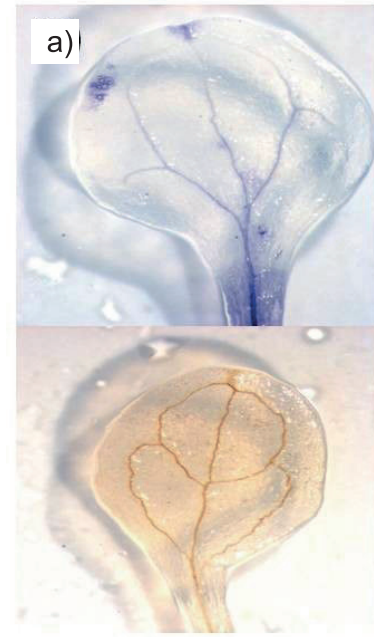

Control

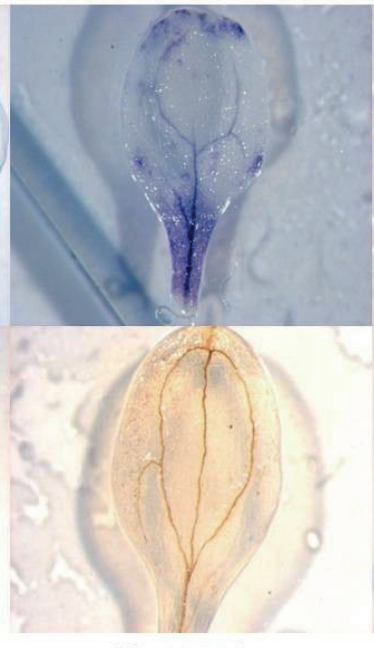

$10 \mu \mathrm{M}$ BPA

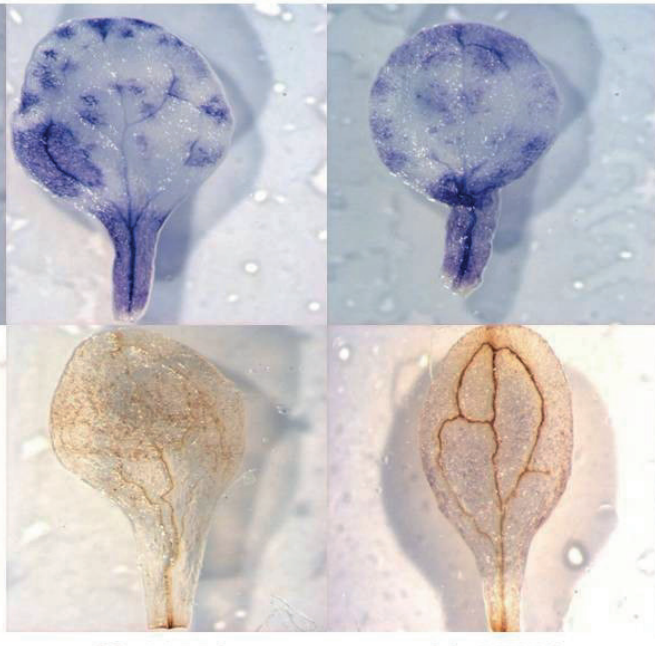

$30 \mu \mathrm{M}$ BPA

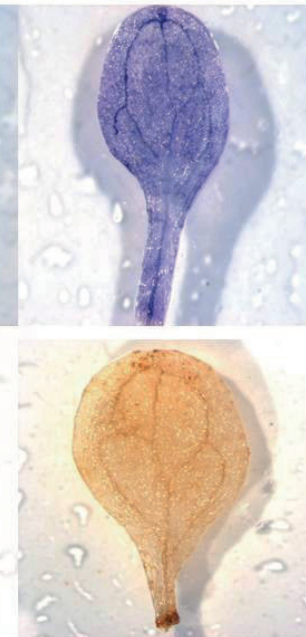

$40 \mu \mathrm{M}$ BPA

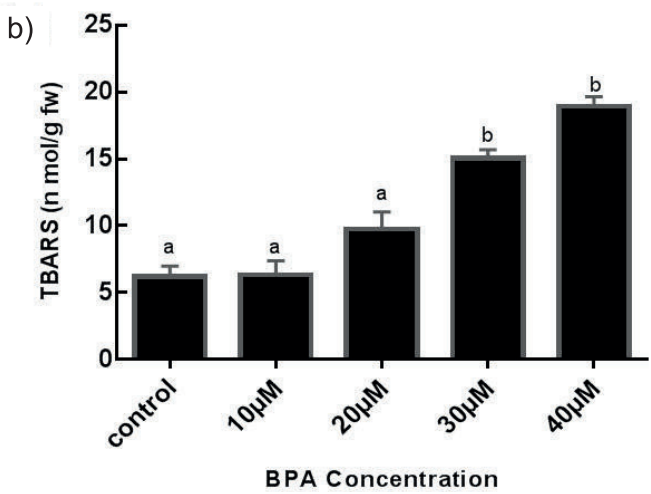

Fig. 5. a) Effect of BPA on ROS accumulation and lipid peroxidation. Brown staining indicates in-situ detection of leaf $\mathrm{H}_{2} \mathrm{O}_{2}$ and blue staining indicates in-situ detection of leaf $\mathrm{O}_{2}^{-}$. b) Effect of BPA on MDA content in leaves of WT. The error bars indicate means \pm SE. Different letters indicate significant differences $(\mathrm{P}<0.05)$.

by modified cellular status. Therefore, the present study was designed to evaluate the effect of BPA on the leaves of hydroponically grown Arabidopsis seedlings. In the present study, we assess the response of the model plant Arabidopsis thialiana to BPA exposure in association with its physiological and biochemical responses and expression of certain chlorophyll synthesis and antioxidant defensive system genes. In addition, the effect of BPA on the ultra-structural morphology of leaf cells and organelle structure was also determined using transmission and scanning electron microscopy.

\section{BPA Inhibits Growth in Arabidopsis}

In ecotoxicology studies, it is imperative to understand the toxicological effects of pollutants on organisms in order to devise and adapt proper management strategies that may reduce the effects of contaminants on key organisms of an ecosystem and minimize the environmental toxicological risks. BPA have obvious detrimental and toxicological effects on plants such as inhibition of root and shoot growth and photosynthesis $[7,29]$. Plant growth status can be best presented by growth indices such as plant height, fresh weight, and leaf area. The obtained results showed that in response to a low concentration of BPA $(10 \mu \mathrm{M})$, the rosettes' fresh weight increased in Arabidopsis seedlings (Fig. 1), and BPA exposure at high concentrations ( 30 and $40 \mu \mathrm{M}$ ) decreased the fresh weight of rosettes. The decrease in fresh weight may be related to BPA-induced inhibition of photosynthesis leaf area expansion, respiration, primary metabolism, ion imbalance, or disturbances in ion homeostasis. Photosynthesis plays a key role in synthesis and accumulation of organic substances necessary for plant growth and development. Thus, the limitation in the photosynthesis of plants might be the main reason that environmental pollutant affects the growth of plants [15]. The decrease in rosette fresh weight of Arabidopsis leaves in response to BPA exposure can also be explained by the net effect of toxicities, such as ROS accumulation and lipid peroxidation.

\section{BPA Exposure Reduces Photosynthetic Pigments in Arabidopsis}

Chlorophyll has a dual function in photosynthesis, both mediating light-driven charge separation and harvesting and transferring light energy to reaction centers [1]. 
a)

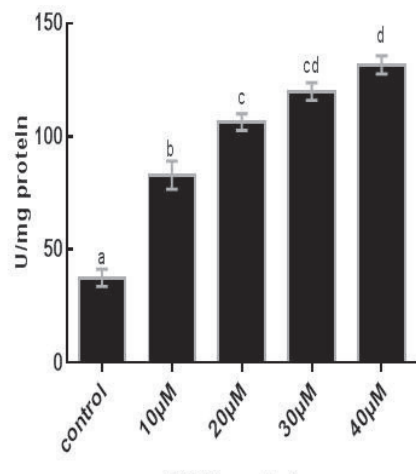

BPA Concentration

CAT activity

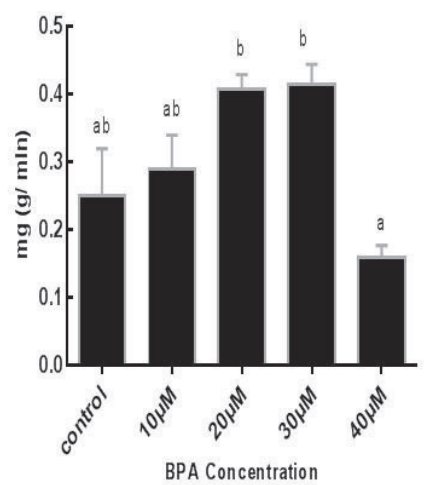

POD activity

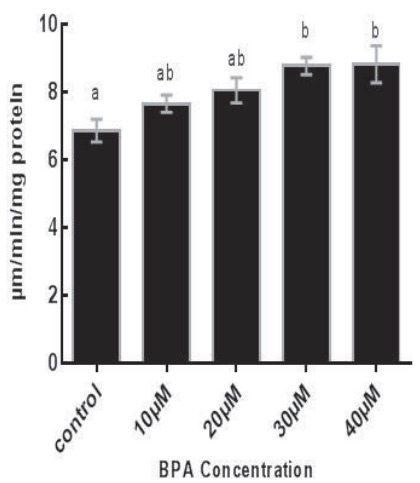

APX activity

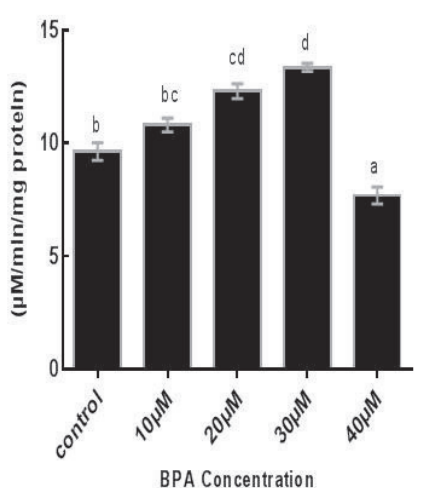

b)

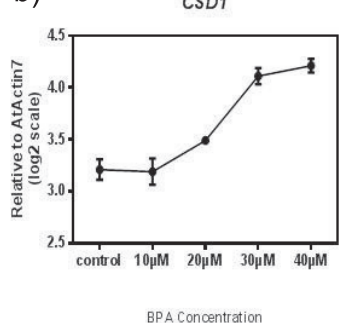

CAT2

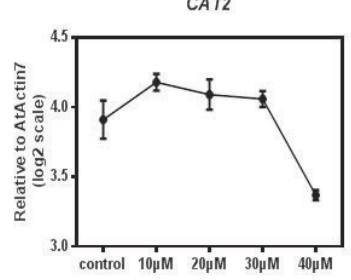

BPA concentration

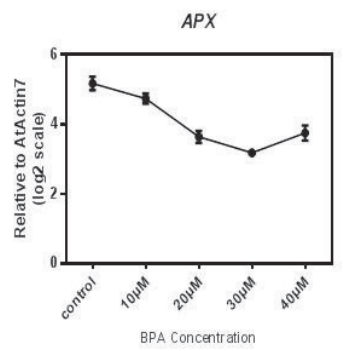

$M S D$

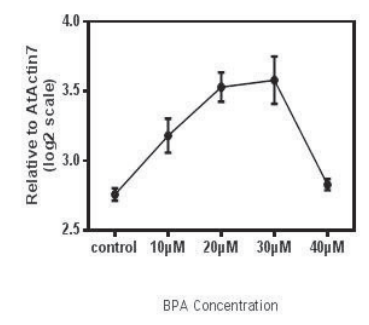

CAT3

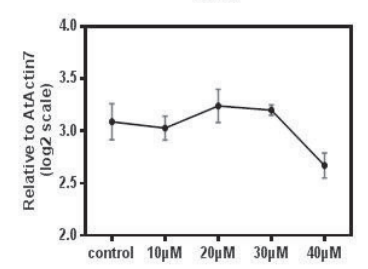

BPA Concentration

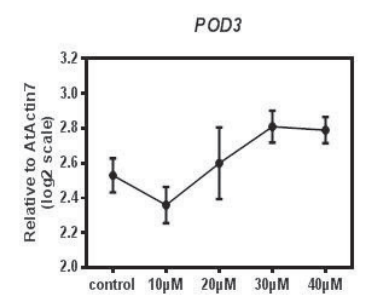

BPA Concentration

Fig. 6. a) Antioxidant enzyme activity in leaves of Arabidopsis $W T$ following BPA treatment. The error bars indicate means \pm SE. Different letters indicate significant differences $(\mathrm{P}<0.05)$. b) Effect of BPA on the transcription level of genes related to antioxidant enzyme activity in leaves of Arabidopsis WT leaves. The transcription levels were normalized with AtActin 7 in each sample, and the expression value was presented in $\log 2$ scale, error bar mean $\pm \mathrm{SD}$.

Thus the pigment content of plant cells directly affects photosynthetic rate, indirectly changing plant quality and yield [12]. In Arabidopsis, upon exposure to BPA all tested pigments (chlorophyll $a$, chlorophyll $b$, and carotenoids) were significantly reduced. It has been repeatedly reported that BPA exposure reduces photosynthesis by causing the destruction of photosynthetic pigments $[2,17]$. Since chlorophyll synthesis is a step-wise process and blockage of any of the five steps involved in chlorophyll synthesis can lead to reduced pigment content [12], we speculate that BPA implicated the synthesis of chlorophyll. The reduced pigments content can also be explained by the peroxidation of chloroplast membrane and disruption of thylakoids in the leaves of Arabidopsis caused by BPA, which was also proven by ultra-structure analysis.

Having shown that chlorophyll content decreased in response to BPA exposure, we wanted to further examine if the expression of the chlorophyll synthesis genes is affected by BPA. Consistent with the results that BPA exposure at higher concentrations significantly reduced chlorophyll content, expression of the chlorophyll synthesis genes also was reduced at higher BPA concentrations.

\section{BPA Exposure Induced Alterations in Organelle Structure}

In Arabidopsis, the control cells of the leaves showed intact chloroplast containing granum, stroma, lamella, and thylakoids. However, in the treated cells BPA not only reduced the number of chloroplasts but also induced damage in the ultrastructure of chloroplasts involving disruption of the thylakoid system and stroma. One of the most important structural units of chloroplasts, the thylakoids plays a key role in photosynthesis. This damage to thylakoids can also be associated with a decrease in photosynthetic activity. The chloroplasts, mitochondria, and peroxisomes are the integral components of ROSproducing machinery; the photosynthetic light reaction in chloroplast, the aerobic respiration in mitochondria, and photorespiration in the peroxisomes generates ROS [8]. The deformed shape of chloroplast may result from their swelling due to a compromised chloroplast envelope, which may affect the chloroplast-thylakoid related processes such as light harvesting, electron transport, and ion movement [30]. The ultra-structural alterations of chloroplasts in BPA-treated leaves might be 
due to an increase in the production of ROS, which can cause oxidative damage to cellular components and their function [5]. Additionally, the decrease in photosynthetic activity can also be associated with chloroplast-thylakoiid damage. The decrease in starch granule count under BPA applications can be correlated with glucose starvation due to reduced photosynthetic activity [28].

In the same way, BPA adversely affected ultrastructure of the nucleus as evident by its disintegrated or irregular appearance. Furthermore, in the BPA-treated leaves the shriveled appearance of the cells may be due to compromised membrane permeability. The ultrastructural damages might be attributed to oxidative stress caused by BPA, which would have damaged membranous structures of organelles by lipid peroxidation.

The number of mitochondria also decreased in BPA treated cells. The morphological alterations of mitochondria are one of the early symptoms of whether cells are affected by ROS [10]. Stress can induce mitochondria swelling by affecting mitochondrial inner membrane permeability. In the treated cells, an increased number of plastoglobuli can be attributed to the protective role of plastoglobuli under BPA stress, which caused oxidative damage to the photosynthetic apparatus [3]. This increase in plastoglobuli count can also be related to higher levels of plastoglobule-specific proteins, which can positively influence the synthesis and accumulation of lipid molecules (carotenoids, tocopherol, and plastoquinone) [14, 24]. It is well known that under stress conditions, these types of lipid molecules act as electron transport carriers and play an important role in protecting the photosynthetic apparatus from oxidative damage [13].

\section{BPA Exposure Damages Stomata and Guard Cells}

In the study on Arabidopsis, SEM analysis revealed alterations in leaf abxial surface and deformed and irregular stomata as a consequence of damaged guard cells. Moreover, SEM micrographs indicated that BPA exposure markedly decrease the number of open stomata. The reduced number of open stomata may be due to the damaged guard cells, resulting in reduced turgor pressure and ultimately leading to the closure of stomatal pores [6]. The damage to the stomatal aperture can be attributed to increased accumulation of ROS in chloroplasts of stomata.

\section{BPA Exposure Induces ROS Production in Rice and Arabidopsis Seedlings}

In plants reactive oxygen species are produced as a byproduct of various metabolic pathways that are localized in different cellular compartments [9, 18]. However, under environmental stresses, excess ROS is produced beyond the scavenging capacity of plants, which can result in the accumulation of ROS beyond the antioxidant scavenging capacity of a plant, thus creating oxidative stress in chloroplasts, plasma membrane, mitochondria, and peroxisomes [26]. TBARS, an efficient biomarker, is used to evaluate the level of oxidative stress in an organism. The damage to the cytoplasmic membrane resulting from various environmental stresses directs the synthesis of MDA through a series of physiological reactions [11]. In the present study, our results showed an increase in the level of $\mathrm{O}_{2}$ - and $\mathrm{H}_{2} \mathrm{O}_{2}$ as well as an increase in MDA content in the leaves of Arabidopsis seedlings. The increase in $\mathrm{O}_{2}$ - and $\mathrm{H}_{2} \mathrm{O}_{2}$ not only corresponded to the increase of MDA in the leaves but also corresponded to the decrease of plant growth and pigment content. Our results exhibited that the increase in BPA concentration aggravates the level of ROS in Arabidopsis. An increased level of ROS can tamper with the polyunsaturated fatty acid in the cell membrane, triggering membrane lipid peroxidation and compromising membrane permeability [1]. Moreover, through Fenton reaction excessive ROS can be used to generate more toxic $\cdot \mathrm{OH}$ [19], which can affect a plant's physiological activities and growth. Since lipid peroxidation can lead to the formation of TBARS, the measurement of ROS like $\mathrm{H}_{2} \mathrm{O}_{2}$ and $\mathrm{OH}^{-}$contents can be used as suitable physiological markers and also as an explanation for MDA increase [4]. In the present study, $\mathrm{H}_{2} \mathrm{O}_{2}$ and $\mathrm{OH}^{-}$contents corresponded well to the pattern of TBARS, i.e., the increase in BPA concentration resulted in a significant increase in the production of ROS (Figs 3.2B-C). These results confirmed that a possible reason for the increased TBARS content and lipid peroxidation in Arabidopsis seedlings is the production of ROS at high concentrations upon exposure to BPA. In the present study, BPA exposure caused excessive accumulation of ROS, leading to the self-catalytic effect of free radical chain reactions that are responsible for decreasing the membrane fluidity and membrane lipid peroxidation. The increased oxidative stress and lipid peroxidation could possibly have caused cell death and declined antioxidant enzyme activities in Arabidopsis.

\section{Response of Antioxidant System to BPA Exposure}

The effect of BPA on the antioxidant system of Arabidopsis leaves was also studied. In response to the adverse effects of BPA, plants have devised complex detoxification mechanisms that consist of both enzymatic and non-enzymatic antioxidants to ensure their growth and development [41]. As the first line of defense against ROS, SOD enzymes play a key role in detoxification of ROS, followed by other key antioxidant enzymes (POD, CAT, and APX) [26]. Our results indicated a strong SOD and POD activity in BPA-treated Arabidopsis leaves. These results were also confirmed by qRT-PCR analysis, which showed that transcript accumulation of CSD1 $(\mathrm{Cu} / \mathrm{Zn}$ $S O D)$ and $P O D 3$ in leaves were progressively stimulated in a dose-dependent manner in response to BPA exposure. However, the transcript level of MSD (MnSOD) showed an increase up to $30 \mu \mathrm{M}$ BPA concentrations, followed by a decrease at the highest tested BPA concentration $(40 \mu \mathrm{M})$. The difference in the responses of MnSOD and 
$\mathrm{Cu} / \mathrm{ZnSOD}$ in plants may be due to their different subcellular locations or upstream sequences in the genome. Moreover, it was noted that CAT and APX activities were more enhanced in Arabidopsis leaves at lower concentrations of BPA (up to $30 \mu \mathrm{M}$ BPA); however, at the highest concentration of BPA $(40 \mu \mathrm{M})$ we observed a considerable decrease in activities of both CAT and APX. The expression analysis of $A P X$ gene showed not direct correlation with the corresponding enzyme activity. The activity of APX enzyme was high at lower concentrations but the APX gene showed a continuous decreasing pattern with increasing concentrations of BPA. The differential response of APX enzyme and $A P X$ gene expression may be due to the presence of different isoforms of antioxidant enzymes [8]. The reduced activities of both CAT and APX in response to the highest BPA concentration indicated an incomplete activation of an antioxidant defensive system and may be the reason for excess ROS accumulation.

\section{Conclusion}

Conclusively, it may be said that reduction in pigment content, accumulation of ROS, and its subsequent damage to organelle structure indicated that BPA induces oxidative stress in Arabidopsis thialiana in a dosedependent manner. The results also showed that the antioxidant defensive system was activated in response to BPA exposure and played a vital role in resistance against BPA stress. However, at high concentrations of BPA $(40 \mu \mathrm{M})$, excess ROS may have accumulated beyond the scavenging capacity of antioxidant enzymes. However, the present study was conducted in hydroponics-based conditions. In order to evaluate the toxicological effects of BPA on plants further research in a soil-based environment is reccommended. In addition to biochemical experiments the use of new approachers such as plant omics will further elucidate the mechanism involved in mediating plant responses to BPA-induced stress.

\section{Acknowledgements}

Our research was funded by the Zhejiang Provincial Natural Science Foundation of China (grant No. LZ15C020001), the National Key R\&D Program of China (No. 2016YFD0100701), the National Natural Science Foundation of China (Nos. 31570183, 31529001, and 31370215), and the Major State Basic Research Development Program (Program 973, grant No. 2015CB150200).

\section{References}

1. ISLAM F., ALI S., FAROOQ M.A., WANG J., GILL R.A., ZHU J., ZHOU W. Butachlor-Induced Alterations in Ultrastructure, Antioxidant, and Stress-Responsive Gene
Regulations in Rice Cultivars. CLEAN - Soil, Air, Water, 1500851, 2017.

2. ATKINSON N.J., DEW T.P., ORFILA C., URWIN P.E. Influence of combined biotic and abiotic stress on nutritional quality parameters in tomato (Solanum lycopersicum). J. Agric. Food Chem., 59 (17), 9673, 2011.

3. ALI I., LIU B.H., FAROOQ M.A., ISLAM F., AZIZULLAH A., YU C.Y., GAN Y.B. Toxicological effects of bisphenol A on growth and antioxidant defense system in Oryza saliva as revealed by ultrastructure analysis. Ecotoxicol. Environ. Saf. 124, 277, 2016.

4. COOPER J.E., KENDIG E.L., BELCHER S.M. Assessment of bisphenol A released from reusable plastic, aluminium and stainless steel water bottles. Chemosphere, 85 (6), 943, 2011.

5. ZHANG J.Z., LI X.Y., ZHOU L., WANG L.H., ZHOU Q., HUANG X.H. Analysis of effects of a new environmental pollutant, bisphenol A, on antioxidant systems in soybean roots at different growth stages. Sci. Rep., 6, 2016.

6. WANG Q., WANG L., HAN R., YANG L., ZHOU Q., HUANG X. Effects of bisphenol A on antioxidant system in soybean seedling roots. Environ. Toxicol. Chem., 34 (5), 1127, 2015.

7. YAMAMOTO T., YASUHARA A., SHIRAISHI H., NAKASUGI O. Bisphenol A in hazardous waste landfill leachates. Chemosphere, 42 (4), 415, 2001.

8. HUNT P.A., SUSIARJO M., RUBIO C., HASSOLD T.J. The Bisphenol A Experience: A Primer for the Analysis of Environmental Effects on Mammalian Reproduction. Biol. Reprod., 81 (5), 807, 2009.

9. KUNDAKOVIC M., CHAMPAGNE F.A. Epigenetic perspective on the developmental effects of bisphenol A. Brain. Behav. Immun., 25 (6), 1084, 2011V

10. SPERONI L., VOUTILAINEN M., MIKKOLA M.L., KLAGER S.A., SCHAEBERLE C.M., SONNENSCHEIN C., SOTO A.M. New insights into fetal mammary gland morphogenesis: differential effects of natural and environmental estrogens. Sci Rep., 7, 40806, 2017.

11. RHEE J.-S., KIM B.-M., LEE C.J., YOON Y.-D., LEE Y.M., LEE J.-S. Bisphenol A modulates expression of sex differentiation genes in the self-fertilizing fish, Kryptolebias marmoratus. Aquat. Toxicol., 104 (3), 218, 2011.

12. VALIPOUR M. Global experience on irrigation management under different scenarios. J. Water L. Dev. 32 (1), 95, 2017.

13. VALIPOUR M. Drought Analysis in Different Basins and Climates. Taiwan Water Conserv., 65 (1), 55, 2017.

14. VALIPOUR M. Future of agricultural water management in Africa. Arch. Agron. Soil Sci., 61 (7), 907, 2015.

15. VALIPOUR M. How much meteorological information is necessary to achieve reliable accuracy for rainfall estimations? Agriculture, 6 (4), 53, 2016.

16. YANNOPOULOS S.I., LYBERATOS G., THEODOSSIOU N., LI W., VALIPOUR M., TAMBURRINO,A., ANGELAKIS A.N. Evolution of water lifting devices (pumps) over the centuries worldwide. Water, 7 (9), 5031, 2015.

17. FLINT S., MARKLE T., THOMPSON S., WALLACE E. Bisphenol A exposure, effects, and policy: A wildlife perspective. J. Environ. Manage., 104, 19, 2012.

18. NAKAJIMA N., OHSHIMA Y., SERIZAWA S., KOUDA T., EDMONDS J.S., SHIRAISHI F., MORITA M. Processing of bisphenol A by plant tissues: Glucosylation by cultured BY-2 cells and Glucosylation/translocation by plants of Nicotiana tabacum. Plant Cell Physiol. 43 (9), 1036, 2002. 
19. PAN W.J., XIONG C., WU Q.P., LIU J.X., LIAO H.M., CHEN W., ZHENG L. Effect of BPA on the germination, root development, seedling growth and leaf differentiation under different light conditions in Arabidopsis thaliana. Chemosphere, 93 (10), 2585, 2013.

20. ZHANG J.Z., WANG L.H., LI M., JIAO L.Y., ZHOU Q., HUANG X.H. Effects of bisphenol A on chlorophyll fluorescence in five plants. Environ. Sci. Pollut. Res., 22 (22), 17724, 2015.

21. FERRARA G., LOFFREDO E., SENESI N. Phytotoxic, clastogenic and bioaccumulation effects of the environmental endocrine disruptor bisphenol $\mathrm{A}$ in various crops grown hydroponically. Planta, 223 (5), 910, 2006.

22. QIU Z., WANG L., ZHOU Q. Effects of bisphenol A on growth, photosynthesis and chlorophyll fluorescence in above-ground organs of soybean seedlings. Chemosphere, 90 (3), 1274, 2013.

23. WEN Y., CHEN H., SHEN C., ZHAO M., LIU W. Enantioselectivity tuning of chiral herbicide dichlorprop by copper: roles of reactive oxygen species. Environ. Sci. Technol. 45 (11), 4778, 2011.

24. KUMAR A., PRASAD M.N.V, MOHAN V., ACHARY M., PANDA B.B. Elucidation of lead-induced oxidative stress in Talinum triangulare roots by analysis of antioxidant responses and DNA damage at cellular level. (n.d.)

25. HAFSI C., ROMERO-PUERTAS M.C., GUPTA D.K., DEL RIO L.A., SANDALIO L.M., ABDELLY C. Moderate salinity enhances the antioxidative response in the halophyte Hordeum maritimum L. under potassium deficiency. Environ. Exp. Bot., 69 (2), 129, 2010.

26. CONN S.J., HOCKING B., DAYOD M., XU B., ATHMAN A., HENDERSON S., GILLIHAM M. Protocol: optimising hydroponic growth systems for nutritional and physiological analysis of Arabidopsis thaliana and other plants. Plant Methods., 9, 2013.

27. AHAMMED G.J., CHOUDHARY S.P., CHEN S.C., XIA X.J., SHI K., ZHOU Y.H., YU J.Q. Role of brassinosteroids in alleviation of phenanthrenecadmium co-contaminationinduced photosynthetic inhibition and oxidative stress in tomato. J. Exp. Bot., 64 (1), 199, 2013.

28. GRATÃO P.L., MONTEIRO C.C., CARVALHO R.F., TEZOTTO T., PIOTTO F.A., PERES L.E.P., AZEVEDO R.A. Biochemical dissection of diageotropica and Never ripe tomato mutants to Cd-stressful conditions. Plant Physiol. Biochem. 56, 79, 2012.

29. THORDAL-CHRISTENSEN H., ZHANG Z., WEI Y., COLLINGE D.B. Subcellular localization of $\mathrm{H}_{2} \mathrm{O}_{2}$ in plants. $\mathrm{H}_{2} \mathrm{O}_{2}$ accumulation in papillae and hypersensitive response during the barley - powdery mildew interaction. Plant J., 11 (6), 1187, 1997.

30. FRYER M.J., OXBOROUGH K., MULLINEAUX P.M., BAKER N.R. Imaging of photo-oxidative stress responses in leaves. J. Exp. Bot. 53 (372), 1249, 2002.

31. DAUD M.K., MEI L., AZIZULLAH A., DAWOOD M., ALI I., MAHMOOD Q., ZHU S.J. Leaf-based physiological, metabolic, and ultrastructural changes in cultivated cotton cultivars under cadmium stress mediated by glutathione. Environ. Sci. Pollut. Res., 23 (15), 15551, 2016.

32. ZHOU W., LEUL M. Uniconazole-induced tolerance of rape plants to heat stress in relation to changes in hormonal levels, enzyme activities and lipid peroxidation. Plant Growth Regul., 27 (2), 99, 1999.

33. NAKANO Y., ASADA K. Hydrogen peroxide is scavenged by ascorbate-specific peroxidase in spinach chloroplasts. Plant Physiol. Biochem., 22 (5), 867, 1981.

34. AEBI H. [13] Catalase in vitro. Methods Enzymol., 105, 121,1984

35. LIU Y., LIU D., HU R., HUA C., ALI I., ZHANG A., GAN Y. AtGIS, a $\mathrm{C} 2 \mathrm{H} 2$ zinc-finger transcription factor from Arabidopsis regulates glandular trichome development through GA signaling in tobacco. Biochem. Biophys. Res. Commun., 483 (1), 209, 2016.

36. LIVAK K.J., SCHMITTGEN T.D. Analysis of relative gene expression data using real-time quantitative PCR and the 2(T)(-Delta Delta C) method. Methods, 25 (4), 402, 2001.

37. TANAKA A., ITO H., TANAKA R., TANAKA N.K., YOSHIDA K., OKADA K. Chlorophyll a oxygenase (CAO) is involved in chlorophyll $\mathrm{b}$ formation from chlorophyll $\mathrm{a}$. Proc. Natl. Acad. Sci., 95 (21), 12719, 1998.

38. LANGE B.M., GHASSEMIAN M. Genome organization in Arabidopsis thaliana: a survey for genes involved in isoprenoid and chlorophyll metabolism. Plant Mol. Biol., 51 (6), 925, 2003.

39. STEPHENSON P.G., TERRY M.J. Light signalling pathways regulating the Mg-chelatase branchpoint of chlorophyll synthesis during de-etiolation in Arabidopsis thaliana. Photochem. Photobiol. Sci., 7 (10), 1243, 2008.

40. WANG X., YANG X., CHEN S., LI Q., WANG W., HOU C., WANG S. Zinc Oxide Nanoparticles Affect Biomass Accumulation and Photosynthesis in Arabidopsis. Frontiers in plant science, 6, 2015.

41. CHEN G., REN L., ZHANG J., REED B.M., ZHANG D., SHEN X. Cryopreservation affects ROS-induced oxidative stress and antioxidant response in Arabidopsis seedlings. Cryobiology, 70 (1), 38, 2015. 